\title{
There Is No Distinctively Semantic Circularity Objection to Humean Laws
}

\author{
David Mark Kovacs \\ Department of Philosophy, Tel Aviv University, Tel Aviv, Israel \\ Email: david.mark.kovacs@gmail.com
}

\begin{abstract}
Humeans identify the laws of nature with universal generalizations that systematize rather than govern the particular matters of fact. Humeanism is frequently accused of circularity: laws explain their instances, but Humean laws are, in turn, grounded by those instances. Unfortunately, this argument trades on controversial assumptions about grounding and explanation that Humeans routinely reject. However, recently an ostensibly semantic circularity objection has been offered, which seeks to avoid reading such assumptions into the Humean view. This paper argues that the new semantic version tacitly relies on the familiar metaphysical one and, therefore, it ultimately brings nothing new to the table.
\end{abstract}

Keywords: Best System Account; circularity objection; grounding; explanation; Humeanism; Laws of Nature; truthmaker semantics

1.

Humean theories of laws contend that ultimately all there is to reality are particular matters of fact (the "Humean mosaic") and that the laws of nature are universal generalizations that merely summarize these facts. ${ }^{1}$ Such views can be contrasted with non-Humean accounts, according to which the laws are something "over and above" universal generalizations that "govern" the particular matters of facts. ${ }^{2}$ These characterizations are intentionally impressionistic; the proper formulation of Humeanism is subject to controversy. Most contemporary Humeans understand the clause "ultimately all there is" in terms of grounding: the laws are grounded in their instances. As we will see, some Humeans reject this characterization of their view. ${ }^{3}$ But in setting up the problem, it will be useful as a natural point of departure.

Humean accounts are frequently accused of a kind of explanatory circularity. According to widespread scientific practice, the objection goes, laws often explain their instances. But Humean laws are supposedly grounded in the mosaic, which includes those instances. So the laws explain and are explained by their instances contrary to the received view that explanation is asymmetric. Call this the Metaphysical Circularity Objection (MCO). ${ }^{4}$ The objection deserves the label 'metaphysical' because it assumes that Humean laws are metaphysically explained by their instances and that metaphysical and scientific explanation can be chained to produce an (in this case circular) explanation.

\footnotetext{
${ }^{1}$ See Lewis (1983, 366-68), Lewis (1986a, ix), Lewis (1986b, 122-25), Lewis (1994, 478-80) Loewer (1996), Beebee (2000), and Schaffer (2008) for influential statements of Humeanism about the laws.

${ }^{2}$ See Dretske (1977), Tooley (1977), Armstrong (1983), and Maudlin (2007).

${ }^{3}$ See Bhogal (2020a) for an overview of Humeanism about the laws, including different ways of formulating it.

${ }^{4}$ See Armstrong $(1983,40)$, Bird (2007, 86), and Maudlin $(2007,172)$.

(C) The Author(s), 2021. Published by Canadian Journal of Philosophy. This is an Open Access article, distributed under the terms of the Creative Commons Attribution licence (http://creativecommons.org/licenses/by/4.0/), which permits unrestricted re-use, distribution, and reproduction in any medium, provided the original work is properly cited.
} 
Humeans have not been swayed by this argument. A common response is that while laws scientifically explain their instances and the instances metaphysically explain the laws, these two types of explanation aren't chainable so as to yield a violation of any asymmetry principle worth caring about. ${ }^{5}$ Other Humeans argue that the laws are not grounded or metaphysically explained by their instances and recommend some alternative way of understanding the slogan that the laws are "nothing over and above" the mosaic. ${ }^{6}$

In light of these responses, a circularity objection that doesn't read grounding-theoretic assumptions into the Humean account of laws would be of considerable interest. Recently, Stefan Roski (2018) and Erica Shumener (2019) have independently developed just such an argument, which goes roughly as follows. Humean laws are special kinds of universal generalizations. But all the information that an instance of a universal generalization contains is part of the information that the generalization contains. Thus, if a Humean law explains its instance then the same explanatory information appears in both the explanans and the explanandum, which makes the explanation circular. Following Shumener, I will call this the Semantic Circularity Objection (SCO). The argument deserves the label 'semantic' because it ostensibly trades on the information content of the putative explanantia and explananda, rather than their grounding status.

Arguments in this ballpark are not entirely new. Roski traces the SCO back to Dretske (1977, 262), while Shumener notes that Hempel and Oppenheim $(1948,162)$ raised and quickly dismissed a similar worry. Thus, the SCO's significance for the debate between Humean and non-Humean accounts of laws goes well beyond the success of Roski's and Shumener's specific arguments. Nonetheless, in this paper I will primarily focus on their versions. Since these are much more detailed than Dretske's and Hempel and Oppenheim's and are explicitly put forth as not relying on the MCO, they demand a serious response from the Humean. However, I will argue that, taken at face value, Roski's and Shumener's versions of the SCO fail to show that Humeans are committed to circular explanations. Moreover, to derive a genuinely circular explanation, they need to add just the kinds of grounding-theoretic assumptions they sought to bypass. This means that, in the end, the SCO doesn't pose any novel challenge to Humeanism over above what it already faces in the form of the MCO.?

In the next section, I will begin with Roski's argument, which uses less technical machinery. In section 3, I will move on to Shumener's version, which relies on the notions of partial content and exact verification from truthmaker semantics. In section 4 , I will conclude that, as of yet, we have been given no distinctively semantic circularity challenge to the Humean approach to laws, and that there is reason to think that no such challenge is forthcoming, either.

\section{2.}

Roski's primary concern isn't the Humean conception of laws per se but the explanatory connection between universal generalizations and their instances. He focuses on the tension between the following two theses $(2018,1986)$ :

(IN-BY-GEN) Some true universal generalizations partially explain their instances

(GEN-BY-IN) Every true universal generalization is partially explained by its instances

\footnotetext{
${ }^{5}$ Loewer (2012), Hicks and van Elswyk (2015), and Bhogal (2020b) defend versions of this response.

${ }^{6}$ See Marshall (2015), Miller (2015) and Kovacs (forthcoming) for such strategies.

${ }^{7}$ Some might think that universal generalizations are conceptually explained/grounded by their instances, but I use 'metaphysical explanation' and 'grounding' broadly enough to cover conceptual explanation and grounding. I assume that Roski and Shumener are similarly inclusive; their claim to having an argument that is independent of the familiar MCO shouldn't depend on fine-grained insider debates in the grounding literature.
} 
Roski proposes to resolve the tension by giving "reasons to reject IN-BY-GEN that are independent of its conflict with GEN-BY-IN" $(2018,1998)$. He begins by defending the following principle:

A.1 There is no true instance of the following scheme:

That $a$ is $F$ and $b$ is $F$ explains why $a$ is $F$. $(2018,1999)$

If $a$ 's being $F$ and $b$ 's being $F$ could explain why $a$ is $F$, Roski argues, we would need to admit objectionably circular and uninformative explanations; hence, A.1 (more on this step in a moment). But if A.1 is true, he continues, so are the following two principles:

A.1' There is no true instance of the following scheme:

That $a$ is $F$ and $b$ is $F$ and $c$ is $F$, and ... ad. inf. explains why $a$ is $F$.

A. $1^{\tau}$ There is no true instance of the following scheme:

That $a$ is $F$ and $b$ is $F$ and $c$ is $F$, and ... ad. inf. and $a ; b ; c ; \ldots$ are all and only the objects there are explains why $a$ is $F$.

As Roski concedes, A.1 doesn't logically entail A.1', nor does A.1' logically entail A.1 ${ }^{\tau}$. Still, it's arbitrary to accept A1 but not A1' and A.1 ${ }^{\tau}$. The only difference between A.1 and A.1' is that the latter concerns infinite conjunctions, and it's unclear why an infinite conjunction could explain its conjuncts any more than a finite one. Likewise for A1' and A. $1^{\tau}$ : if infinite conjunctions cannot explain their conjuncts, why should it help to amend them with a totality clause? Finally, an infinite conjunction amended with the corresponding totality clause logically entails a universal generalization. Moreover, Roski adds, the generalization logically entails the conjunction of each of its instances $(2018,1999)$. Although this close logical relationship between universal generalizations and their instances is consistent with universal generalizations explaining their instances even assuming A. $1^{\tau}$, Roski thinks that we would need a compelling reason to drive a wedge between infinite conjunctions amended with a totality clause and universal generalizations. Having found no such reason, he concludes that (IN-BY-GEN) is false. At this point, the problem for Humeans is obvious. Humean laws are universal generalizations, so if (IN-BY-GEN) is false, they cannot explain their instances. ${ }^{8}$

Yet the argument doesn't show what it's supposed to. I will grant the move from A.1 to A. $1^{\tau}$ and won't worry about the move from conjunctions to universal generalizations. Let's focus instead on the very first step, A.1. Why accept it? Roski starts by reminding us that explanation is irreflexive: no proposition figures among its own explanantia. For example, $<$ Socrates is a philosopher $>$ cannot explain $<$ Socrates is a philosopher $>$. But then, he reasons, $<$ Socrates is a philosopher and Kant is a philosopher $>$ cannot explain $<$ Socrates is a philosopher $>$, either. $<$ Kant is a philosopher $>$ is explanatorily irrelevant to <Socrates is a philosopher $>$, so conjoining it with < Socrates is a philosopher> yields an "explanation" that is not only circular but even contains irrelevant information. The reasoning generalizes: a putative explanation of $\mathrm{A}$ by $\mathrm{A}$ is circular, and the conjunction of A with some further proposition, B, at best adds explanatorily irrelevant extra information but still leaves us with a circular explanation.

Now, I agree with Roski about this particular example: <Socrates is a philosopher and Kant is a philosopher $>$ doesn't explain <Socrates is a philosopher $>$. However, this doesn't show that no conjunction can explain its conjuncts. For one, there are respectable views on which some explanations proceed from complex entities to their constituents. For example, in the neoAristotelian tradition integrated wholes are often taken to be explanatorily prior to their arbitrary

\footnotetext{
${ }^{8}$ Roski suggests a few ways to explain away the intuition that some universal generalizations explain their instances (2018, 2000-2). One of them is that although no universal generalization explains its instances, laws do. This strategy is unavailable to Humeans, though, since Humeans think that laws are universal generalizations (of a special sort).
} 
proper parts. ${ }^{9}$ (Jonathan Schaffer [2010] also relies on this intuition in one of his arguments for priority monism.) Integrated wholes are in an important sense more complex than their parts. This needs to be understood in the right way: in the fundamentality hierarchy, integrated wholes come before their parts and, in that sense, are "simpler". But in the mereological sense they are more complex than things to which they are explanatorily prior. If this is so, explanations that go from logically complex propositions to logically simpler ones should no longer appear so outlandish, either. For another, and more to the point, there are defensible cases of a conjunction explaining its conjunct. Any such case is bound to be controversial since, given the asymmetry of explanation, it will be in tension with the popular principle that conjunctions are grounded by their conjuncts (more on which below). To my mind, the most priming cases are "top down" explanations in which the collective behavior of a system explains the behavior of its members. Take, for example, a school of fish that swims in the direction of food. By 'school' I don't mean a further entity over and above the fishes that compose it; I simply mean fish $f_{1}$, fish $f_{2}, \ldots$, fish $f_{\mathrm{n}}$ taken together, when their behavior is coordinated in a certain way. In that case, the fact that the school swims in the direction of the food is plausibly identical to the fact that $f_{1}$ swims in the direction of the food $\& f_{2}$ swims in the direction of the food $\& \ldots \& f_{n}$ swims in the direction of the food. (Note that we cannot identify the school's swimming with the plurality of facts that consists of $f_{1}$ 's, $f_{2}$ 's, etc. swimming, for this would mean that one fact is identical to many facts taken together. So even though the school is a plurality of fishes, its behavior is the conjunction rather than the plurality of the individual fishes' behavior.) Now, for many, and perhaps all, individual members, the fact that the school swims in the direction of food explains why that particular member swims in the direction of the food. That is, $f_{1}$ swims in the direction of the food because the school does. But given our identification of the school's swimming with the conjunction of $f_{1}$ 's, $f_{2}$ 's, $\ldots$, and $f_{n}$ 's swimming, this means that $f_{1}$ swims in the direction of the food because $f_{1}$ does $\& f_{2}$ does $\& \ldots \& f_{n}$ does. That is, a conjunction explains one of its conjuncts. ${ }^{10}$

While I find this example persuasive enough, I don't pretend that it's irresistible. I'm especially aware that any such example of a conjunction explaining its conjuncts will go against popular principles about grounding (in particular Conjunction Grounding, which I will get to in a moment), and I don't expect grounding theorists already set in their ways to give them up on the basis of one counterexample. But when considered without theoretical prejudice, the example does seem quite plausible. (A few paragraphs on I will also argue that Humeans in particular should not shun such explanations.) Moreover, cases of conjunctions explaining their conjuncts don't by themselves violate the irreflexivity of explanation without some supplementary assumption.

This last point is worth belaboring. Circularity is normally understood to require either self- or mutual explanation: a partial explanation of $\varphi$ by $\psi$ is circular if and only if either $\varphi=\psi$ or $\varphi$ partially explains $\psi$ (here and throughout I use 'partial explanation' inclusively, so as to cover full explanation as a limiting case). On the assumption that partial explanation is distributive, we can get an extended definition of "one-many" circularity: a partial explanation of $\varphi$ by a set of propositions $\Gamma$ is circular if and only if some member of $\Gamma$ (including possibly $\varphi$ itself) is among $\varphi$ 's explanantia. ${ }^{11}$ However, the distributivity of partial explanation doesn't entail that a putative explanation of some proposition A by a proposition that involves $\mathrm{A}$ as a conjunct is also circular, since $\mathrm{A}$ is not a member of the set of propositions whose only element is $\mathrm{A} \& \mathrm{~B}$. Things would be different with a putative explanation of A

\footnotetext{
${ }^{9}$ Two recent examples are Inman (2018) and Koslicki (2018).

${ }^{10}$ For a different example, see Kovacs $(2020,1674)$.

${ }^{11}$ That partial explanation is distributive means that if $\varphi \in \Gamma$ and $\wedge \Gamma$ partially explain $\psi$, then $\varphi$ partially explains $\psi$. Distributivity also follows from a natural definition of partial in terms of full explanation, according to which $\varphi$ partially explains $\psi$ if and only if there is a set of propositions $\Gamma$ such that $\varphi \in \Gamma$ and $\wedge \Gamma$ fully explain $\psi$. (This is a generalization of the standard definition of partial grounding to all kinds of explanation; see, e.g., Dixon [2016,376] for this definition and note 4 on the same page for further references). Leuenberger (2020, 2655-56) questions the standard definition on the basis that some facts might have partial but not full grounds [cf. also Fine 2012, 53], but his arguments don't affect the distributivity of partial explanation.
} 
by $A$ and $B$ taken together. That would be circular, since $A$ is an element of the set of propositions, $\{\mathrm{A}$, $B$, whose members supposedly explain $A \cdot{ }^{12}$ Roski notes that $A$ is a "part of" $A \& B$, but this is just another way of saying that $A$ is a conjunct of $A \& B$. And given that $A \& B$ is not a set of propositions that includes A but a numerically distinct proposition, invoking it to explain A doesn't constitute a formal violation of the irreflexivity of explanation. So anyone who doesn't already accept that conjunctions never explain their conjuncts can reasonably demand an argument for accepting that they never do.

Roski also offers the following supplementary argument to show that conjunction-to-conjunct explanations are unacceptable $(2018,1998-99)$. On pain of circularity, A cannot explain A. Now take some other proposition, $B$. If $B$ is explanatorily independent of $A$ (neither A nor B is among the other's explanantia), it a fortiori doesn't explain A. But the conjunction of two propositions, neither of which helps explain A, cannot itself explain A. If, alternatively, B isn't explanatorily independent of $A$ (i.e., if one of $A$ and B helps explain the other), their conjunction still cannot explain A because it contains irrelevant information. After all, A by itself already contains all the information that is relevant to $A$, thus, any information contained by $B$ that outstrips $A$ is not relevant to A. Either way, A\&B cannot explain A.

I don't find this reasoning persuasive. Focus on the case when the two conjuncts are explanatorily independent. It's plausible that if propositions $\varphi$ and $\psi$ are both explanatorily irrelevant to a proposition $\chi$, then $\varphi$ and $\psi$ together are also explanatorily irrelevant to $\chi$ (though see footnote 11). But from the fact that the individual conjuncts of a conjunctive proposition don't partially explain an explanandum it doesn't follow that the conjunction itself doesn't. Roski reasons that A\&B cannot explain A because neither of its conjuncts "helps explain" A. But the notion of partial explanation (which Roski expresses with the 'helps explain' locution) is normally understood to apply to members of a set or plurality of jointly full explanantia. It doesn't license the inference that a conjunction of propositions (which is a singular candidate explanans, not a set or plurality thereof) can help explain a proposition only by way of the conjuncts "contributing" to the explanation. That is a substantive assumption to the effect that explanatory irrelevance is closed under conjunction.

Humeans not only aren't compelled to accept this assumption but, in fact, have reason to reject it. For on at least one plausible construal of the view, Humean explanations are supposed to unify. ${ }^{13}$ Take the Best System Account (BSA), one of the best worked out version of Humeanism about the laws. According to the BSA, the laws are those universal generalizations that occur as axioms in that axiomatization of our scientific theories that achieves the best trade-off between simplicity and strength. From a Humean perspective, the best systematization is explanatory in a holistic way insofar as it doesn't derive its explanatory power from parts of that systematization. A systematization, $S$, may be superior to another systematization, $S^{*}$, even though the part of $S$ that systematizes propositions $p_{1} \ldots p_{n}$ would by itself be less explanatory than the part of $S^{\star}$ that systematizes $p_{1} \ldots$ $p_{n}$. Moreover, nothing in the Humean view rules out the even more radical possibility that $S$ is an explanatory systematization, even though components of it are by themselves not explanatory at all. If we adopt a winner-take-all conception of explanation, according to which explanation is an allor-nothing matter rather than a matter of degree (and a conception to which unificationists in particular are plausibly committed anyway), ${ }^{14}$ such scenarios are all but guaranteed to occur.

\footnotetext{
${ }^{12}$ Saucedo (2013) has recently questioned the distributivity of partial explanation (see also Bohn 2012). If he is right, there could even be a plurality of propositions $\mathrm{A}_{1} \ldots \mathrm{A}_{n}$ that explain $\mathrm{A}_{i}(i \in\{1 \ldots n\})$ without $\mathrm{A}_{i}$ being a partial explanans of itself and thus without violating the irreflexivity of partial explanation (understood along Saucedo's lines). Clearly, though, this wouldn't help Roski's argument. By granting the distributivity of explanation, my goal is only to emphasize that even if this principle is true, it's entirely silent on the explanatory relation between logically complex propositions and their atomic constituents.

${ }^{13}$ For unificationism about scientific explanation, see Friedman (1974) and Kitcher (1989); and for applications to metaphysical explanation, see Kovacs (2020) and Baron and Norton (2021).

${ }^{14} \mathrm{Cf}$. Woodward (2003, 367-69). Woodward mentions this feature as a disadvantage of unificationism, but it's worth noting that in the realm of both semantic and metaphysical explanations (which are what's at issue here) the winner-take-all conception seems to be the default view.
} 
While BSA theorists aren't committed to full-blown unificationism about explanation, the criteria of when a system achieves the best trade-off between simplicity and strength are similar to the criteria of explanatoriness on unificationist views. So, BSA theorists are in a good position to maintain that explanation is holistic and that an explanatory systematization doesn't have to explain an explanandum by virtue of its members being partial explanantia of that explanandum. A "systematization" is most naturally conceived of as a plurality of propositions, but if a plurality of propositions can be a full explanans without each member of it being a partial explanans, it's also plausible that a conjunction can be a full explanans without each conjunct of it being a partial explanans. ${ }^{15}$

I can think of one more reason for holding that conjunctions never explain their conjuncts, which Roski mentions in passing $(2018,1999$ n41) but doesn't rely on in his subsequent reasoning: that conjunctions are grounded in and therefore explained by their conjuncts ("Conjunctive Grounding"). ${ }^{16}$ Conjunctive Grounding is widely accepted among grounding theorists, and adding it to the negation of A.1 does yield a circular explanation. Indeed, I expect many readers to have scratched on the margins already a few paragraphs ago that "obviously" conjunctions are grounded and explained by their conjuncts and that therefore, given the asymmetry of explanation, they can never explain their conjuncts.

However, appealing to Conjunctive Grounding is illegitimate in the present context, and it's no accident that Roski doesn't officially rely on it. Doing so would constitute an appeal to a groundingtheoretic principle and would mean that Roski's ostensibly semantic argument tacitly appeals to the MCO. Don't object, as many have in conversation, that Conjunctive Grounding is an independently plausible principle. Although, for the reasons explained above, I don't accept Conjunctive Grounding, the principle is so deeply entrenched in the grounding literature that I don't expect anyone to give it up in light of the remarks I made above. The main problem with Conjunctive Grounding is not that it's implausible, but that it's the wrong kind of principle on which to base the SCO. ${ }^{17}$ Recall: the whole point of introducing the SCO was to block Humean strategies that target the groundingtheoretic assumptions of the MCO. Once it turns out that we need to appeal to Conjunctive Grounding in order to justify a crucial premise of the SCO, we lose just this rationale. For at that point, the Humean can pull one of her familiar moves to address the objection: she can argue that the type of explanation conjunctions bear to their conjuncts (the one at issue in Humean laws that explain their instances) differs from the type the conjuncts bear to the conjunction (the one licensed by Conjunctive Grounding), or even eschew grounding-theoretic ideology altogether. Whether Conjunctive Grounding is independently plausible is neither here nor there; what matters is only that if Roski needs to presuppose it then his version of the SCO doesn't give Humeans any reason to change their minds that is independent of the MCO.

\section{3.}

Though Shumener construes explanation as a relation between sentences, her first gloss on the SCO is otherwise similar to Roski's: that part of a universal generalization's content that is identical to its instance's content cannot explain that instance (on pain of violating the noncircularity of explanation), and conjoining it with explanatorily irrelevant content cannot turn it into an explanation either. However, Shumener attempts to make the relevant notion of semantic circularity more

\footnotetext{
${ }^{15}$ See Kovacs (forthcoming, sec. 4) for a similar line of thought.

${ }^{16}$ In fact, the transitivity of explanation and Conjunctive Grounding jointly entail the principle that if $\varphi$ and $\psi$ don't help explain $\chi$ then $\varphi \& \psi$ doesn't help explain $\chi$, either. For suppose that $A \& B$ helps explain $C$ and that $A$ and B help explain A\&B. By transitivity, A and B help explain C. By conditionalization and contraposition, if A and B don't help explain C then A\&B doesn't help explain C, either. Generalizing from the arbitrariness of A, B and C, it follows that explanatory irrelevance is closed under conjunction.

${ }^{17}$ Although for some pushback against Conjunctive Grounding, see Kovacs (2020) and McSweeney (2020).
} 
precise by cashing it out in terms of partial content. The notion of partial content is intuitive: A's content is part of A\&B's content; the content of 'A marble ball is in the philosophy department' is part of the content of 'A bowl of marble balls is in the philosophy department'; etc. Partial content is more fine-grained than asymmetric entailment; for example, AvB's content is not a part of A's content. Following Fine, Shumener $(2019,799)$ defines partial content as follows:

$\mathrm{C}$ is part of the content of $\mathrm{A}$ iff:

(1) Every possible state that exactly verifies A contains a possible state that exactly verifies $C$

(2) Every possible state that exactly verifies $C$ is contained in a possible state that exactly verifies $\mathrm{A}$

Exact verification resists definition in simpler terms. But it's a central notion of Fine's truthmaker semantics, and Shumener also relies on it when developing her argument. ${ }^{18}$ Similarly to partial content, exact verification is a more fine-grained counterpart of the verification relation of possibleworld semantics. For instance, in possible-world semantics any world in which grass is green verifies 'Grass is green.' But in truthmaker semantics, not every state that contains the state [grass is green] is an exact verifier of 'Grass is green.' For example, [grass is green and the sky is blue] isn't, since intuitively the blueness of the sky has nothing to do with the truth of this sentence. The following principles (which both Fine [2017, 562] and Shumener [2019,799] accept) give us a better grasp of the core notion:

(1) State $[s]$ exactly verifies $A \& B$ iff $[s]$ is the fusion of a state [s1] that exactly verifies A and a state [s2] that exactly verifies $B$

(2) State [s] exactly verifies AvB iff [s] exactly verifies A or [s] exactly verifies B

(3) State [s] exactly verifies $\sim$ A iff [s] exactly falsifies A

Shumener $(2019,794)$ argues that universal generalizations explaining their instances would falter on the following constraint:

$\mathrm{CON}$ : If the content of a sentence $\mathrm{E}$ is part of the content of a set of sentences $\Gamma$, then an explanation of $\mathrm{E}$ in terms of $\Gamma$ is unsuccessful ${ }^{19}$

As we will see below, Shumener has more to say in support of CON. But it's worth stating explicitly what the significance of her argument against Humean accounts of laws is. Unlike with the classic MCO, she writes,

"[T]he Humean cannot avoid this semantic circularity by avoiding certain metaphysical relations or distinguishing between scientific and metaphysical explanation. There is only scientific explanation at work here. The claim that the [explanantia] of Humean explanations contain their explananda as part of their content rests on only semantic notions." $(2019,802)$

Yet just like Roski's version of the SCO, Shumener's argument fails to establish its intended conclusion as originally stated and, once appropriately amended, tacitly relies on the familiar MCO. To see why, take a closer look at CON. Why accept it? Shumener defends it as follows. First, explanation is irreflexive: no sentence can help explain itself. Second, explanations shouldn't contain irrelevant information. $\mathrm{CON}$ is then justified on the basis of these two principles: E cannot

\footnotetext{
${ }^{18}$ In an appendix, Shumener offers a modified version of her argument reframed in terms of Stephen Yablo's possible-world semantics for partial content. But when interpreted against this background, her argument suffers essentially from the same difficulty that I will raise below. Moreover, given the possible-worlds framework's limited ability to provide an adequate semantics of grounding (due to the coarser grain of worlds), this modified argument might not even have the fix I will later suggest for the version based on truthmaker semantics.

${ }^{19}$ Shumener's formulation was printed with a typo, which I fixed.
} 
explain E itself, therefore any content that has E's content as a part contains some information that isn't itself explanatorily relevant to E.

For reasons familiar from our discussion of Roski's argument, the irreflexivity of explanation doesn't by itself entail CON. This makes the second step, that "if a set of sentences $\Gamma$ fully explains another sentence, $\mathrm{E}$, then $\Gamma$ is wholly relevant to $\mathrm{E}$, namely, all of the content of the sentences of $\Gamma$ is relevant to explaining E” $(2019,796)$, crucial for Shumener's argument. She unpacks the condition that $\Gamma$ be wholly relevant to $E$ as the condition that the contents of the sentences of $\Gamma$ be explanatorily relevant to $\mathrm{E}$. So the notion of explanatory relevance at issue is a relation between a set of sentence contents and a sentence (or perhaps its content). But why think that A\&B's content cannot bear this relation to A, or that a universal generalization's content cannot bear it to its instances?

We get no straightforward answer to this question from Shumener's discussion. Granted, in truthmaker semantics an exact verifier of A\&B isn't an exact verifier of A. But it doesn't follow, without supplementary assumptions, that an exact verifier of A\&B cannot be explanatorily relevant to A. Similarly, it's unquestionably true that in truthmaker semantics whatever counts as an exact verifier of $\forall x(F x \supset G x)$ isn't an exact verifier of Ga. But an extra premise is needed to infer that an exact verifier of $\forall x(F x \supset G x)$ is explanatorily irrelevant to Ga. Since Shumener explicitly notes that "the exact verification relation is not [...] a metaphysically explanatory relation" $(2019,803)$, it's unclear why the structural constraints on explanatory relevance should be read off the structural constraints on exact verification. So Humeans can reasonably demand an argument for Shumener's tacit assumption that failure of exact verification entails explanatory irrelevance and, for the reasons I mentioned in the previous section, they even have independent reasons to reject this assumption.

As with Roski's version, there is a way to back up the argument. Fine $(2012,72)$ puts truthmaker semantics in the service of offering a semantics of various ground-theoretic notions, as follows (the ' 'symbol stands for fusion, i.e. ' $\left[s_{1} \cdot s_{2}\right]$ ' reads as 'the fusion of $\left[s_{1}\right]$ and $\left[s_{2}\right]$ '. I changed the notation slightly to make it consistent throughout the paper):

$\mathrm{A}_{1}, \mathrm{~A}_{2}, \ldots$ is a weak full ground for $\mathrm{C}$ iff $\left[s_{1} . s_{2} . s_{3} \ldots.\right]$ verifies $\mathrm{C}$ whenever $\left[s_{1}\right]$ verifies $\mathrm{A}_{1},\left[s_{2}\right]$ verifies $\mathrm{A}_{2}$, $\left[s_{3}\right]$ verifies $\mathrm{A}_{3} \ldots$

$A$ is a weak partial ground for $C$ iff for some sentences $A_{1}, A_{2}, \ldots$ (and assignment of verification sets to them) $A, A_{1}, A_{2}, \ldots$ is a weak full ground for $C$

$A_{1}, A_{2}, \ldots$ is a strict full ground for $C$ iff $A_{1}, A_{2}, \ldots$ is a weak full ground for $C$ and $C$ is not a weak partial ground for any of $A_{1}, A_{2}, \ldots$

$\mathrm{A}$ is a strict partial ground for $\mathrm{C}$ iff $\mathrm{A}$ is a weak partial ground for $\mathrm{C}$ but $\mathrm{C}$ is not a weak partial ground for $\mathrm{A}$

We can see that, with these semantic clauses, Fine explicitly presupposes a tight connection between grounding and verification. Moreover, conjunctions and their conjuncts clearly satisfy the righthand side of Fine's clause for strict full ground, since the fusion of the exact verifiers of a conjunction's conjuncts is an exact verifier of the conjunction (but not vice versa). ${ }^{20}$ The case of universal generalizations is more involved, but we need not get into the details here. Shumener discusses two candidate verifiers of a universal generalization's instances: either the exact verifiers of a material conditional's consequent and the exact verifiers of the negation of its antecedent or only the former. But she emphasizes that on either proposal, the fusion of the exact verifiers of a universal generalization's instances, along with a totality state, is itself an exact verifier of that generalization

\footnotetext{
${ }^{20} \mathrm{Or}$, at least, conjunctions that don't consist of multiple occurrences of the same conjunct satisfy the clause. But I take it that it's these conjunctions that are relevant to the argument.
} 
$(2019,800)$. This is all I need to make my point. Observe that Shumener's view meshes with Fine's $(2012,73)$ own semantic clause for universal generalizations (' $\tau$ ' stands for a totality fact):

$[s]$ verifies $\forall x \mathrm{~B}(x)$ iff there are $\left[s_{1}\right],\left[s_{2}\right], \ldots$ such that $[s]=\left[\tau \cdot s_{1} . s_{2} \ldots.\right]$, and $\left[s_{1}\right]$ verifies $\mathrm{B}\left(\mathrm{a}_{1}\right)$, $\left[s_{2}\right]$ verifies $\mathrm{B}\left(\mathrm{a}_{2}\right), \ldots$ (with $\mathrm{a}_{1}, \mathrm{a}_{2}, \ldots$ running through all of the individuals)

Read together with Fine's clauses for weak full, weak partial, and strict full ground, it follows that universal generalizations are (strictly and fully) grounded by their instances plus a totality fact, and that therefore each instance of a universal generalization is a strict partial ground of that generalization. Once we amend Shumener's argument with this last claim, we can justify her tacit assumption that exact verification goes hand in hand with explanatory relevance. In the conjunction case, the failure of $A \& B$ 's exact verifiers to exactly verify $A$ implies that $A \& B$ is not even a weak partial ground of, and is therefore explanatorily irrelevant to, A. Likewise for $\forall x(F x \supset \mathrm{G} x)$ and Ga: the former cannot help explain the latter because an exact verifier of the former isn't an exact verifier of the latter.

Let's be clear about what the problem is with Shumener's argument (and what it isn't). The problem is not that $(\mathrm{CON})$ is implausible. As with Roski's version of the SCO, one might simply insist that $\mathrm{CON}$ is independently plausible, or that at least the premises Shumener cites in its favor (including the second one, that explanations cannot contain irrelevant information) are plausible. But this amounts to little more than saying that Humeanism itself is implausible, since Humeans cannot be expected to accept CON without argument. Why not? Because given the overall Humean picture of explanation, Humeans have no reason to grant that semantic relevance (as characterized in truthmaker semantics and as invoked in the second premise of Shumener's argument for CON) coincides with explanatory relevance (as would be needed to get a genuine case of explanatory circularity).

This is the gap that could be bridged by invoking Fine's grounding-theoretic clauses. But it's just these clauses that Shumener cannot appeal to in order to justify the move from failure of exact verification to failure of explanatory relevance because the whole point of introducing the SCO was to avoid relying on such grounding-theoretic assumptions. In the end, then, her version of the SCO suffers from the same difficulty as Roski's. Taken at face value, it fails to show that the Humean is saddled with a truly circular explanatory structure. And the most natural ancillary assumption that could help us derive this conclusion wheels in precisely the kind of grounding-theoretic machinery that Shumener sought to avoid by developing the objection as a semantic one.

\section{4.}

Roski and Shumener should be commended for trying to develop an enhanced circularity objection to Humeanism that doesn't take for granted the metaphysical assumptions that Humeans routinely reject when responding to the MCO. But a closer look reveals that neither version of the SCO succeeds at showing that explanations by Humean laws are circular. Moreover, once we add the premise needed to derive an instance of circular explanation, the argument is no longer independent of MCO.

The immediate conclusion is that, as of yet, we have no distinctively semantic circularity challenge to Humean accounts of laws. But there is reason for pessimism about the prospects of any argument along similar lines. Any such argument needs to make an inference from the informational asymmetry between a universal generalization and its instances to the circularity of an explanation of the latter by the former. This inference is unwarranted without a substantive principle that links the informational asymmetry in question to an explanatory asymmetry. But every known principle of this sort is grounding-theoretic; there are no plausible explanatory principles about quantified sentences and their instances outside of grounding-theoretic metaphysics. Appeals to the systematic explanatory posteriority of universal generalizations to their 
instances lose their force once we stipulate that grounding-theoretic principles are not in play. For this reason, I suspect that any version of SCO that relies on general explanatory principles about universal generalizations and their instances will ultimately have to fall back on just the sorts of grounding-theoretic principles that we wanted to avoid when we were looking for an alternative to the familiar MCO.

It gets even worse: a case can be made that Humeans have special reasons to reject the semantic principles underlying the SCO even independently of their connection to the MCO. As I argued in section 2, the best-developed version of Humeanism, the BSA, can be motivated by an appeal to unification. Understood along the lines of Kitcher (1989), unification is a matter of complicated trade-offs between formal aspects of simplicity and strength (which are determined by syntactic criteria) and desiderata that are tied to the subject matter of a group of putative explanations (Kitcher calls these formal and nonformal aspects of "stringency"). But the fact that the latter are even relevant to unification makes it extremely unlikely that explanation by unification obeys any narrowly formal principles. In other work (Kovacs 2020), I use this observation to argue that there is no such thing as an "impure logic of ground" (Fine 2012). But the same point also applies to the formal principles that Roski and Shumener invoke in their arguments. If this is along the right lines, then Humeans can not only resist Roski's and Shumener's purportedly semantic versions of the SCO but are also in a good position to anticipate future iterations of the argument.

Throughout the paper, I have focused on the SCO and have carefully avoided taking a stance on the MCO. This is because my goal has been only to defend a conditional claim: if the Humean has an answer to the MCO, then she has an answer to the SCO too. The SCO stands or falls with the MCO and doesn't pose any new challenge to Humean theories of lawhood. In recent years, the most common answer to the MCO has been to distinguish between different types of explanation, such as metaphysical and scientific, and maintain that laws scientifically explain their instances but are metaphysically explained by them. ${ }^{21}$ A more radical approach is to deny that laws are metaphysically, or perhaps in any way, explained by their instances. (Of course, these "groundless Humeans," as Miller (2015) calls them, need to give an alternative account of the sense in which the laws are "nothing over and above" the Humean mosaic. ${ }^{22}$ ) And there are some further possibilities in logical space; for example, Humeans can altogether deny the irreflexivity of explanation and deny that there is any problem with the circularity that the Humean account of laws appears to engender. ${ }^{23}$ My sympathies lie with Groundless Humeanism, but for the purposes of this paper we don't need to choose between these strategies here. It's enough to observe that if the SCO indeed tacitly relies on the same kinds of grounding-theoretic principles as the MCO, then each of these answers can do double duty as an answer to the SCO. Mainstream Humeans can insist that CON is plausible when understood as a thesis about metaphysical explanation, but not as a thesis about scientific explanation (which is what laws bear to their instances); while groundless and anti-irreflexivist Humeans can simply reject the principle wholesale. Mutatis mutandis for Roski's A. $1^{\tau}$.

Where does this leave us? The debate over the MCO is far from being settled, and each of the strategies mentioned above is controversial. But my goal in this paper wasn't to defend any of them. I'm content to have made a case that if they cannot deal with the SCO, that's only because they already cannot deal with the MCO to begin with. In other words, there is no distinctively semantic circularity objection to Humeanism about the laws, only the old (metaphysical) circularity challenge that the semantic versions were invoked to supersede.

\footnotetext{
${ }^{21}$ See Loewer (2012), Hicks and van Elswyk (2015) and Bhogal (2020b) for responses of this type.

${ }^{22}$ Miller (2015) merely considers Groundless Humeanism without outright endorsing it. Other groundless Humeans include Marshall (2015) and Kovacs (forthcoming).

${ }^{23}$ Philosophers who reject the irreflexivity of grounding or explanation include Bliss (2014), Correia (2014), and RodriguezPereyra (2015).
} 
Acknowledgments. Many thanks to Stefan Roski and Erica Shumener for helpful comments on and discussions about previous versions of this paper, and for patiently helping me better understand their views and arguments. In addition, I thank an audience at the workshop "Derivativeness and Priority" at the Università della Svizzera italiana and a number of anonymous referees. Research on this paper was funded by the Israel Science Foundation (grant no. 2035/19), whose support is gratefully acknowledged.

David Mark Kovacs is a senior lecturer of philosophy at Tel Aviv University, Israel. His main area of specialization is cotemporary metaphysics. He also has interests in epistemology, the philosophy of mind, and the philosophy of science.

\section{References}

Armstrong, David M. 1983. What Is a Law of Nature? Cambridge: Cambridge University Press.

Baron, Sam, and James Norton. 2021. "Metaphysical Explanation: The Kitcher Picture." Erkenntnis 86: 187-207.

Beebee, Helen. 2000. "The Non-Governing Conception of Laws of Nature.” Philosophy and Phenomenological Research 61: 571-94.

Bhogal, Harjit. 2020a. "Humeanism about the Laws of Nature." Philosophy Compass 15 (8): e12696.

Bhogal, Harjit. 2020b. "Nomothetic Explanation and Humeanism about Laws of Nature." Oxford Studies in Metaphysics 12: 164-202.

Bird, Alexander. 2007. Nature's Metaphysics: Laws and Properties. Oxford: Clarendon Press.

Bliss, Ricki. 2014. "Viciousness and Circles of Ground." Metaphilosophy 45: 245-56.

Bohn, Einar Duener. 2012. "Monism, Emergence, and Plural Logic.” Erkenntnis 76: 211-23.

Correia, Fabrice. 2014. "Logical Grounds." Review of Symbolic Logic 7: 1-29.

Dixon, T. Scott. 2016. “Grounding and Supplementation.” Erkenntnis 81: 375-89.

Dretske, Fred. 1977. "Laws of Nature.” Philosophy of Science 44: 248-68.

Fine, Kit. 2012. "Guide to Ground.” In Metaphysical Grounding: Understanding the Structure of Reality, edited by Fabrice Correia and Benjamin Schnieder, 37-80. Cambridge: Cambridge University Press.

Fine, Kit. 2017. “Truthmaker Semantics.” In A Companion to the Philosophy of Language, edited by Bob Hale, Crispin Wright, and Alexander Miller, 556-77. Chichester, UK: Wiley.

Friedman, Michael. 1974. "Explanation and Scientific Understanding.” Journal of Philosophy 71: 5-19.

Hempel, Carl G., and Paul Oppenheim. 1948. "Studies in the Logic of Explanation.” Philosophy of Science 15: 135-75.

Hicks, Michael Townsen, and Peter van Elswyk. 2015. "Humean Laws and Circular Explanation.” Philosophical Studies 172: 433-43.

Inman, Ross D. 2018. Substance and the Fundamentality of the Familiar. New York: Routledge.

Kitcher, Philip. 1989. "Explanatory Unification and the Causal Structure of the World." In Scientific Explanation, edited by Philip Kitcher and Wesley Salmon, 410-505. Minneapolis: University of Minnesota Press.

Koslicki, Kathrin. 2018. Form, Matter, Substance. Oxford: Oxford University Press.

Kovacs, David Mark. 2020. "Metaphysically Explanatory Unification.” Philosophical Studies 177: 1659-83.

Kovacs, David Mark. Forthcoming. "The Oldest Solution to the Circularity Problem for Humeanism about the Laws of Nature.” Synthese.

Leuenberger, Stephan. 2020. “The Fundamental: Ungrounded or All-Grounding?” Philosophical Studies 177: $2647-69$.

Lewis, David K. 1983. "New Work for a Theory of Universals." Australasian Journal of Philosophy 61: 343-77.

Lewis, David K. 1986a. Philosophical Papers Vol. 2. Oxford: Oxford University Press.

Lewis, David K. 1986b. “A Subjectivist's Guide to Objective Chance.” In Lewis 1986a, 83-132.

Lewis, David K. 1994. "Humean Supervenience Debugged.” Mind 103: 473-90.

Loewer, Barry. 1996. "Humean Supervenience." Philosophical Topics 24: 101-27.

Loewer, Barry. 2012. "Two Accounts of Laws and Time." Philosophical Studies 160: 115-37.

Marshall, Dan. 2015. “Humean Laws and Explanation.” Philosophical Studies 172: 3145-65.

Maudlin, Tim. 2007. The Metaphysics within Physics. Oxford: Oxford University Press.

McSweeney, Michaela. 2020. "Debunking Logical Ground: Distinguishing Metaphysics from Semantics." Journal of the American Philosophical Association 6: 156-70.

Miller, Elizabeth. 2015. “Humean Scientific Explanation.” Philosophical Studies, 172: 1311-32.

Rodriguez-Pereyra, Gonzalo. 2015. “Grounding Is Not a Strict Order.” Journal of the American Philosophical Association 1: 517-34.

Roski, Stefan. 2018. “Grounding and the Explanatory Role of Generalizations.” Philosophical Studies 175: 1985-2003.

Saucedo, Raul. 2013. "Collective Allism." Unpublished manuscript. Accessed July 8, 2020. https://www.dropbox.com/s/ x2mzamwjf6im7ur/collective_allism-stable_draft.pdf.

Schaffer, Jonathan. 2008. "Causation and the Laws of Nature: Reductionism.” In Contemporary Debates in Metaphysics, edited by Theodore Sider, John Hawthorne, and Dean W. Zimmerman, 82-107. Oxford: Blackwell. 
Schaffer, Jonathan. 2010. "Monism: The Priority of the Whole." Philosophical Review 119: 31-76.

Shumener, Erica. 2019. "Laws of Nature, Explanation, and Semantic Circularity." British Journal for the Philosophy of Science 70 : 787-815.

Tooley, Michael. 1977. "The Nature of Laws." Canadian Journal of Philosophy 7: 667-98.

Woodward, James. 2003. Making Things Happen: A Theory of Causal Explanation. Oxford: Oxford University Press.

Cite this article: Kovacs, D. M. 2021. There Is No Distinctively Semantic Circularity Objection to Humean Laws. Canadian Journal of Philosophy 51: 270-281, doi:10.1017/can.2021.13 